\title{
Efficient NC Process Scheme Generation Method Based on Reusable Macro and Micro Process Fusion
}

\section{Bo Huang ( $\sim$ bo.huang@siat.ac.cn )}

Shenzhen Institutes of Advanced Technology Chinese Academy of Sciences

Kai He

Shenzhen Institutes of Advanced Technology Chinese Academy of Sciences

Rui Huang

HoHai University

Feifei Zhang

Shenzhen Institutes of Advanced Technology Chinese Academy of Sciences

\section{Xiuling Li}

Zhengzhou Railway Vocational \& Technical College

Shusheng Zhang

Northwestern Polytechnical University

\section{Research Article}

Keywords: NC process reuse, macro process, multi-source, NC process fusion

Posted Date: September 24th, 2021

DOI: https://doi.org/10.21203/rs.3.rs-922612/v1

License: (1) This work is licensed under a Creative Commons Attribution 4.0 International License. Read Full License

Version of Record: A version of this preprint was published at The International Journal of Advanced Manufacturing Technology on February 24th, 2022. See the published version at https://doi.org/10.1007/s00170-022-08959-x. 


\title{
Efficient NC process scheme generation method based on reusable macro and micro process fusion
}

\author{
Bo Huang ${ }^{1,2,3^{*}}$, Kai He ${ }^{1,2,3}$, Rui Huang ${ }^{4}$, Feifei Zhang ${ }^{1,2,3}$, Xiuling $\mathrm{Li}^{5}$, Shusheng Zhang ${ }^{6}$ \\ (1 Shenzhen Institute of Advanced Technology, Chinese Academy of Sciences, Shenzhen 518055, China \\ 2 Shenzhen Key Laboratory of Precision Engineering, Shenzhen 518055, China \\ 3 Guangdong-Hong Kong-Macao Joint Laboratory of Human-Machine Intelligence-Synergy Systems, Shenzhen \\ Institute of Advanced Technology, Chinese Academy of Sciences, Shenzhen, 518055, China \\ 4 College of IOT Engineering, HoHai University, Changzhou 213022, China \\ 5 Zhengzhou Railway Vocational \& Technical College, Zhengzhou, 450052, China \\ 6 School of Mechanical Engineering, Northwestern Polytechnical University, Xi'an, 710072, China)
}

\begin{abstract}
Process reuse technology has been widely studied and applied in manufacturing industry. However, the current $\mathrm{NC}$ process reuse generally assumes that the micro process is compatible with the macro process, but in fact, the reusable processes from the similar local structures of multiple parts are usually difficult to be compatible with each other under the overall manufacturing requirements of the query parts, which leads to the fact that a large amount of user interactions are still required for modification and adjustment in practical engineering applications, so it is not significant to improve the design efficiency. Therefore, an efficient NC process scheme generation method based on reusable macro and micro process fusion is proposed in this paper. Firstly, according to the calculation of semantic distance of process design intention, the micro processes are mapped to the macro process to realize the fusion of the macro process and the micro process, and a compatibility credibility evaluation model is established to evaluate the compatibility of fusion results. Then, when the fusion result is credible, the machining areas corresponding to the process scheme are adjusted and optimized from the geometric level. The adjustment and optimization of machining areas mainly realize the integration of machining areas and the optimization of machining sequence. Finally, the effectiveness and feasibility of the proposed method are verified by the test of actual parts.
\end{abstract}

Key words: NC process reuse; macro process; multi-source; NC process fusion

\section{Introduction}

In recent years, driven by the emergence of cloud computing, big data, the development of the Internet of things and other new generation artificial intelligence technologies, and the deep integration with advanced manufacturing technology, a new generation of intelligent manufacturing technology has been derived, which has triggered major and profound changes in the production mode, manufacturing mode, design mode and other aspects of the manufacturing industry [1,2]. So far, the new generation of intelligent manufacturing, which is mainly characterized by the new generation of artificial intelligence technology, has received a lot of research and application in academia and industry, and a number of new research and application hotspots such as Internet of things, cloud manufacturing and digital twin have emerged [3]. Process design is the key link of product manufacturing, and intelligent process design system is also one of the core research contents of intelligent manufacturing. At the same time, a large number of 3D CAD models and their associated NC process information are constantly generated

\footnotetext{
* Corresponding author: Bo Huang

E-mail address: bo.huang@siat.ac.cn
} 
and accumulated in enterprises, and these data contain rich experience and knowledge of designers [4]. Among them, the most effective utilization method of these process data is process reuse. A variety of process reuse technologies have been adopted in manufacturing enterprises to support the improvement of process design efficiency, such as group technology-based process reuse [5], template-based process reuse [6-9] and case-based process reuse [10,11].

However, in the current process design, the application of NC process reuse mainly focuses on the micro process level, while human interaction design pays more attention to the macro process level. In the research process, it is found that the existing bottom-up process design methods usually imply the assumption that the micro process is compatible with the macro process However, because reusable processes have the characteristics of multi-source (from multiple parts), heterogeneous (heterogeneity of manufacturing resources, difference of process parameters, etc.), local (feature level or local structure level), it is usually difficult to accommodate reusable processes, micro processes and macro processes, resulting in many breakpoints and adjustment/integration requirements. Therefore, user interaction is usually required to eliminate the possible heterogeneity and incompatibility, which leads to the fact that NC process reuse does not significantly improve the efficiency of the overall process design of parts. Therefore, it is urgent and important to study the effective fusion and compatibility evaluation of NC processes of similar local structures.

On the other hand, through the continuous iterative integration and evaluation of multi-source macro process and micro process, the initial NC process scheme can be quickly generated. However, only from the semantic and parameter levels, there are still problems of non-optimization of machining area and machining sequence. Generally speaking, the machining scheme needs to follow the principle of making full use of the machining capacity of the cutting tool, that is, a cutting tool should process all the areas that can be machined in place as far as possible. In addition, the machining area of the same cutting tool also needs to be "one size fits all" as far as possible to reduce cutting lifting. Therefore, it is necessary to study the process optimization and adjustment of machining area fusion and machining sequence optimization.

Based on the above background and requirements, an efficient $\mathrm{NC}$ process scheme generation method based on reusable macro and micro process fusion is proposed in this paper. Firstly, according to the principle of $\mathrm{NC}$ process reuse and the process decision-making behavior of designers, the principle of similar NC process fusion of multi-source local structure is analyzed. Then, the compatibility evaluation method of micro process and macro process fusion is proposed to realize the reusable $\mathrm{NC}$ process fusion, so as to quickly generate the initial $\mathrm{NC}$ process scheme and evaluate the compatibility. Finally, under the guidance of macro process, the machining effectiveness of each manufacturing feature under macro process is analyzed. According to the principle of making full use of cutting tool machining ability, the machining areas are integrated, the machining sequence is adjusted, and "one size fits all" is realized as far as possible, so as to realize the fusion and optimization of NC process.

\section{Related work}

\subsection{NC process reuse}

So far, many research results of process reuse have been widely used in manufacturing industry. Jong et al. [12] proposed an automatic process planning method based on machining feature recognition and group technology. Firstly, the manufacturing features of parts are 
recognized, and then according to the basic attributes of parts (such as part type, material, etc.) and the recognized machining features, the parts are coded by group technology, and the corresponding process procedures are found from the typical process library. However, this method is grouped by part code, and the granularity of process reuse is relatively extensive. Recognizing the above problems, Zheng et al. [13] proposed a process design method based on multi granularity process knowledge. Firstly, the process knowledge is divided into four levels: typical process route, typical feature process chain, typical working procedure and typical working step, and then multi-level process knowledge reuse is realized by group technology. However, because group technology coding can not completely describe the information and process knowledge of parts, and has strong subjectivity and difficult to expand, the retrieved information is extensive and usually only suitable for reference reuse. Chen et al. [14] first interactively selected and adjusted the predefined macro process scheme template, and then guided by the macro process scheme, applied reasoning knowledge to determine the machining methods, cutting tools and cutting parameters of features to build a NC machining unit, Finally, the serialized machining units are mapped to the $\mathrm{NC}$ process chain to realize the $\mathrm{NC}$ process design driven by macro process scheme. Li et al. [15] proposed a dynamic feature information model for NC process design of aircraft structural parts. The method adaptively establishes the evolution state of each feature according to the typical macro process scheme template selected interactively, and then makes decisions on the involved machining parameters. Although the above methods have achieved good results in improving the efficiency and quality of process planning, they still remain "strongly dependent" on expert knowledge and experience; In addition, its core is a structured process knowledge base (including heuristic rule base, process scheme template base, feature base, etc.), which needs to be constructed interactively by experienced process designers, so its degree of automation is low, and its flexibility, scalability, and optimization are poor. Huang et al. [16][17] proposed a multi granularity retrieval method of 3D CAD model for NC process reuse, which provides a new support means for the fine reuse of $\mathrm{NC}$ process with similar parts/features. However, most of the current process reuse methods follow the "1-nearest neighbor criterion", that is, only the process instances with the highest similarity in the retrieval results are used, and the process plan is changed to meet the processing requirements of the query geometry, which leads to the ineffective utilization of multi-source process schemes. Considering these, Xu et al. [18] used the grey correlation analysis method to obtain the adaptability of NC process values of similar instances relative to query features, so as to support the fusion of NC process elements of similar instances to generate NC process schemes with query features. However, this method does not integrate the macro process, and is easy to fall into local optimization, so the practicability of the generated process scheme is still not strong.

\subsection{Automatic process adaptation}

In recent years, with the emergence of multi-variety and small-batch production modes in enterprises, design changes are becoming more and more frequent, which puts forward new requirements for adaptive changes. On the other hand, in the common case-based design method, designers first retrieve the appropriate existing design instance in the instance library according to the current design requirements, and then modify the instance to meet the new design requirements [19]. With the continuous breakthrough of research results related to design reuse, the attention of adaptive change is also growing. Due to the distributed and event-driven characteristics of function block, Wang et al. [20-22] applied function block to distributed process 
design for the first time. Function block data and time-driven trigger mechanism are used to sense changes in the machine tool and production status through its monitoring function to support the adjustment of the NC process by triggering the function block. Wang et al. [23] proposed a NC tool path generation method based on feature and agent drive to quickly respond to the dynamic changes of parts and $\mathrm{NC}$ process in the manufacturing process. In this work, when the feature changes, the feature is activated by agents to make an appropriate response to change the machining parameters, and then automatically update the NC tool path in the CAM system. Therefore, this method has certain "autonomy" and "intelligence". Liu et al. [24] proposed a feature-based adaptive NC programming method for the uncertain demand of manufacturing resources. $75 \%$ of the programming work unrelated to manufacturing resources is completed by the preprocessing module, and the remaining $25 \%$ is completed by the post-processing module. The programming flexibility is realized by dividing the NC programming process into two modules: preprocessing and post-processing. Aiming at the problems of high planning complexity and low efficiency in the process planning of part variant, Qing et al. [25] proposed a configurable machining process planning method for part family, which comprehensively applies the feature-based part variant model and process configuration mechanism. The traditional process design system for a single model is upgraded to a design system that can process a group of parts (i.e. part family), which effectively improves the flexibility and adaptability of the process design system. In general, the above method essentially improves the adaptability of the process design system from the macro level of response mechanism, design mode, etc. Although they can improve the efficiency of $\mathrm{NC}$ process changes when manufacturing resources are uncertain, they cannot support for process reuse-oriented adaptation, mainly because process reuse adaptation needs to solve the multi-source and heterogeneous problems of reusable processes, which are mainly adjusted and optimized for local, micro, and specific NC processes.

\section{Principle of reusable process fusion of multi-source similar parts}

The fusion principle of reusable process fusion of multi-source similar parts is mainly analyzed from two aspects: the fragmentation and heterogeneous characteristics of reusable NC process, and the comprehensive behavior and solution mechanism of designers in the process of process decision-making.

For reusable NC processes fusion of multi-source similar parts, their fragmentation characteristics of the reusable processes derived from content-based retrieval technology are often multi-source, heterogeneous and partial. Although they can be refined, they lack the consideration of the compatibility between multi-source, heterogeneous and local reusable process segments and the process context (process elements related to the current process). On the contrary, the ability of global control and associative memory reasoning possessed by experienced process designers is lacking in computers at present. In fact, according to the theory of cognitive science, process designers also use rule reasoning (semantic memory reasoning) and case reuse (situational memory reasoning), which also have the characteristics of fragmentation, but people can often find the semantic association and breakpoint connection path between fragmented process knowledge at a higher level. The means used include semantic association analysis, context awareness, importance analysis, etc. and the effective integration of multi-source, heterogeneous and fragmented processes is realized through the iterative process of top-down and bottom-up.

Specifically, reusable process fusion of multi-source similar parts mainly deals with two parts: (a) aiming at the semantic gap (heterogeneity) between macro process and micro process, that is, 
there are non-isomorphic working steps between macro process and micro process (such as working steps $W S_{1}$ and $W S_{2}$ in Fig. 1), According to the semantic association analysis of process design intention, it is necessary to establish the association relationship of isomorphic working steps (with the same process design intention) between macro process and micro process to evaluate their compatibility (such as isomorphic working steps $W S_{3}, W S_{4}, W S_{5}$ and $W S_{6}$ identified with the same color circle in Fig. 1); (b) In view of the heterogeneity between micro processes, such as the inconsistency of process parameters associated with isomorphic working steps (such as machining resources, machining allowance, cutting parameters, etc.), its compatibility needs to be evaluated according to the process context and machining geometry of the features. For the isomorphic working steps $W S_{3}, W S_{4}$ and $W S_{5}$ in Fig. 1, if the machining allowance is inconsistent, but they are mapped to the same working step $W S_{6}$ of the macro process, they need to be set uniformly.

Based on the above analysis, the reusable process fusion of multi-source similar parts can be summarized into two aspects: fusion and compatibility evaluation of micro process and macro process, and adjustment and optimization of machining process, as shown in Fig. 1. Specifically:

(1) Compatibility evaluation of fusion result of micro process and macro process

Firstly, the compatibility evaluation needs to map the micro processes to the macro process in order to quickly generate the initial NC process scheme. The basic idea is to transform the problem into the similarity evaluation of process design intention and the overall matching between macro process and micro process. Then, according to the matching results of the whole $\mathrm{NC}$ process scheme and the semantic similarity of design intention, a compatibility credibility evaluation model is constructed.

(2) Adjustment and optimization of machining process

According to the compatibility evaluation results, the similar NC process fusion results are divided into credible and incredible. When it is incredible, it indicates that the micro process is incompatible with the macro process, and it needs to be re-decision and iterative fusion; On the contrary, based on the initial NC process scheme generated by fusion, the machining geometry such as machining area and residual area is calculated, so as to support the subsequent adaptive adjustment of machining geometry, machining tools and machining parameters of isomorphic working steps from different features in the same working step, so that the multi-source, heterogeneous, and fragmented micro processes and macro process are integrated into a complete process situation. 


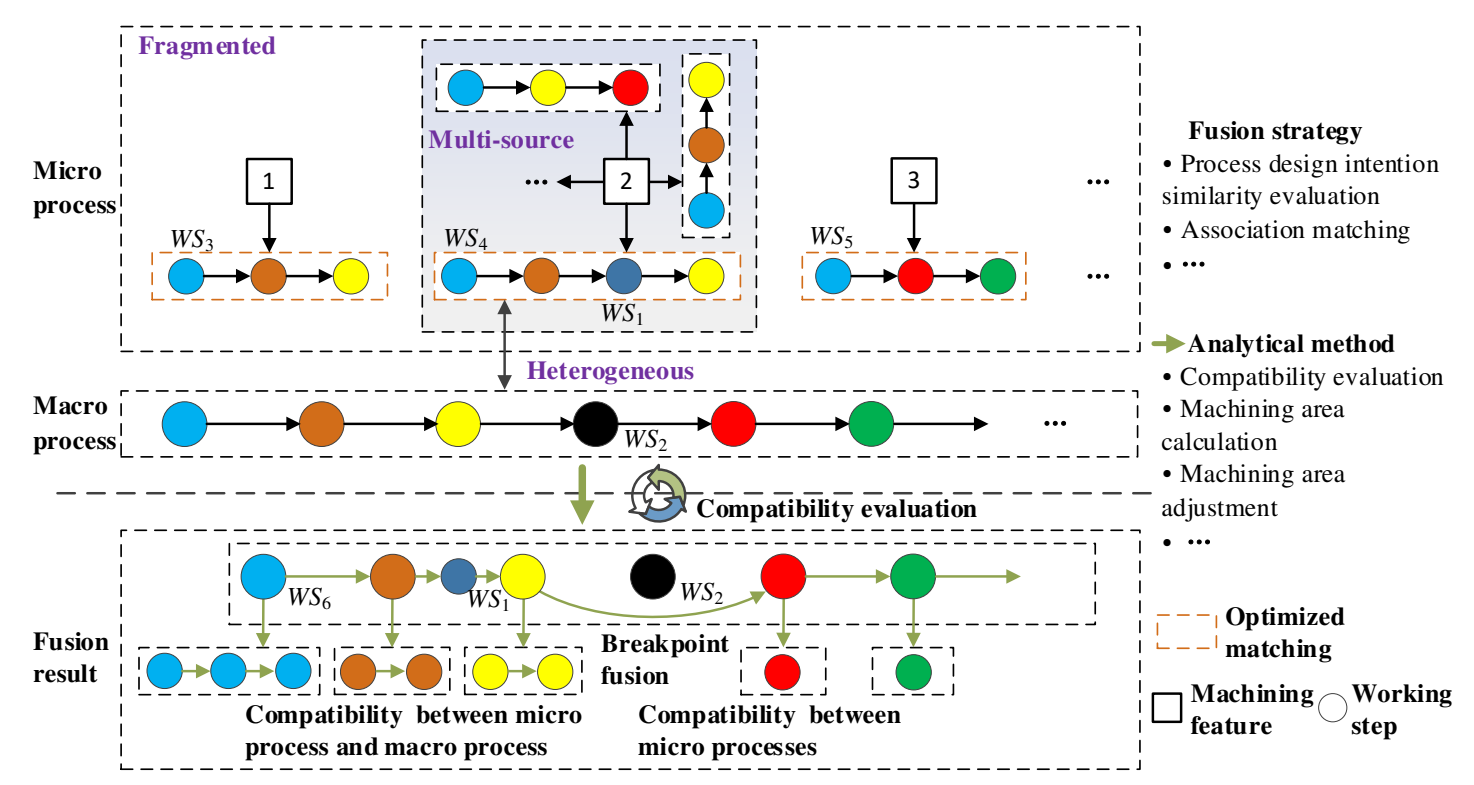

Fig. 1 Principle of reusable process fusion of multi-source similar parts

\section{Compatibility evaluation of fusion result of micro process and macro process}

The mapping from micro process to macro process is actually to calculate the semantic distance of process design intention between macro process and micro process, and realize the overall matching process between macro process and micro process. In this way, the overall idea of the mapping from micro process to macro process is as follows: firstly, the macro process and micro process are expressed as a set of working steps and machining operations respectively, and expressed and described by semantics; Then, the machining operation/working step based on semantic representation is regarded as a node endowed with attributes, and the attributes of the node indicate the information of the design intention of the element. In this way, a macro process/micro process can be represented by a group of attribute nodes, and the fusion between micro process and macro process can be realized by finding the optimal matching between the two groups of nodes; Finally, the Kuhn-Munkres algorithm of complete bipartite graph is used to realize the optimal matching between the two groups of nodes, so as to realize the mapping from micro process to macro process. Therefore, the mapping from micro process to macro process is mainly divided into three parts: (1) process information representation based on semantics; (2) similarity evaluation of process design intention; (3) overall matching of macro process and micro process. According to the matching results, a compatibility credibility evaluation model can be established.

\subsection{Process information representation based on semantics}

In order to facilitate the application of macro process, the reusable macro process $M A P$ of query parts in this paper is mainly composed of working step $W S_{i}$ with temporal relationship, and the working procedures and working orientations are regarded as a part of working step attributes. The reusable micro process $M I P(F)$ of query parts is composed of the machining operation sequence $o p_{k j}$ of features. It can be expressed as follows: 


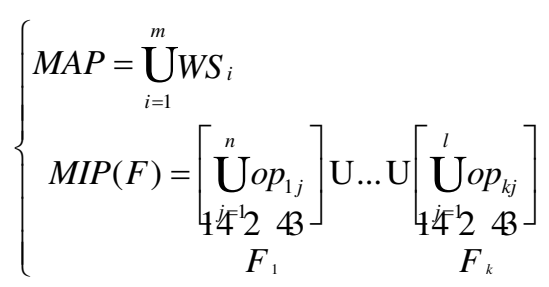

Where, working step $W S_{i}$ includes working step name, machining allowance, cutting tool, working orientation and other information. In order to match the micro process with the macro process, the related features, cutting tools, driving geometric offset and machining coordinate system are mainly applied in the machining operation $o p_{k j}$.

It can be seen from the above that the matching process between the machining operations in the reusable micro process and the working steps in the reusable macro process is actually the analysis process of the process design intention of the machining operation. Since the macro process information is mainly expressed by semantics, in order to analyze the design intention of machining operations based on semantics, firstly, the information used for matching machining operations or working steps is organized through semantic expression, and then the instantiated semantic fragments are constructed by concepts and attributes to describe the process information to be matched.

The semantic information of one machining operation or working step to be matched includes concepts, attributes, relationships and instantiation information. The above organizational structure can be stored in the form of a graph, which is formally expressed as $K E O=\left(K E_{-} I D, C T\right.$, $A T, R T$ ), where:

$K E_{-} I D$ represents the unique identifier of the machining operation or working step to be matched;

$C T$ is the concept table, which stores the concept node and its instantiation information in the machining operation or working step semantic graph;

$A T$ is the attribute table, which stores the attribute node and its instantiation information in the machining operation or working step semantic graph, as well as the associated concept node;

$R T$ is the relation table, which stores the relation information between concepts in the machining operation or working step semantic graph.

The storage structure diagram of the semantic information description of the specific machining operation or working step is shown in Fig. 2.

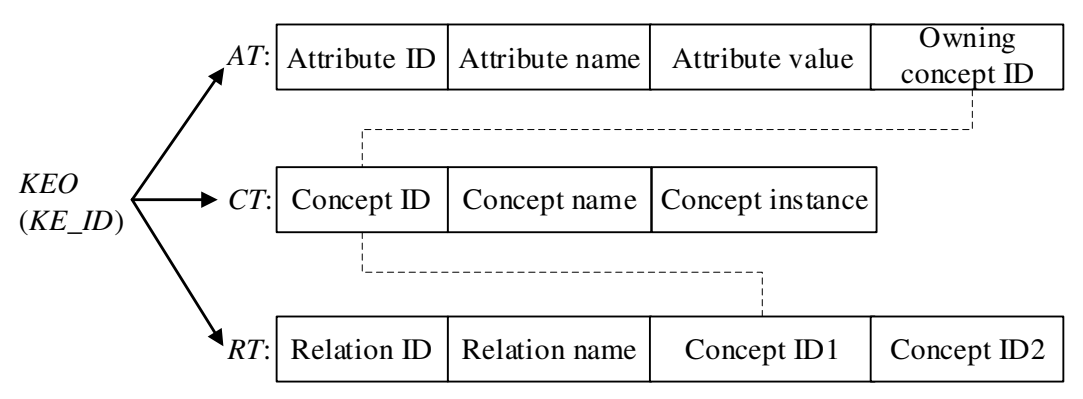

Fig. 2 Storage diagram of machining operation/working step semantic information description

Take two machining operations in an aircraft structure as an example, one rough machining operation and one finish machining operation. The instantiated storage fragments describing these two machining operations are shown in Fig. 3. CID1 and CID2 respectively represent the concept segments of the two operations stored in CT. AID1 is the tool diameter attribute segment stored in 
$A T$, which belongs to CID1, while RID1 is the priority relationship segment between CID1 and CID2 stored in $R T$.

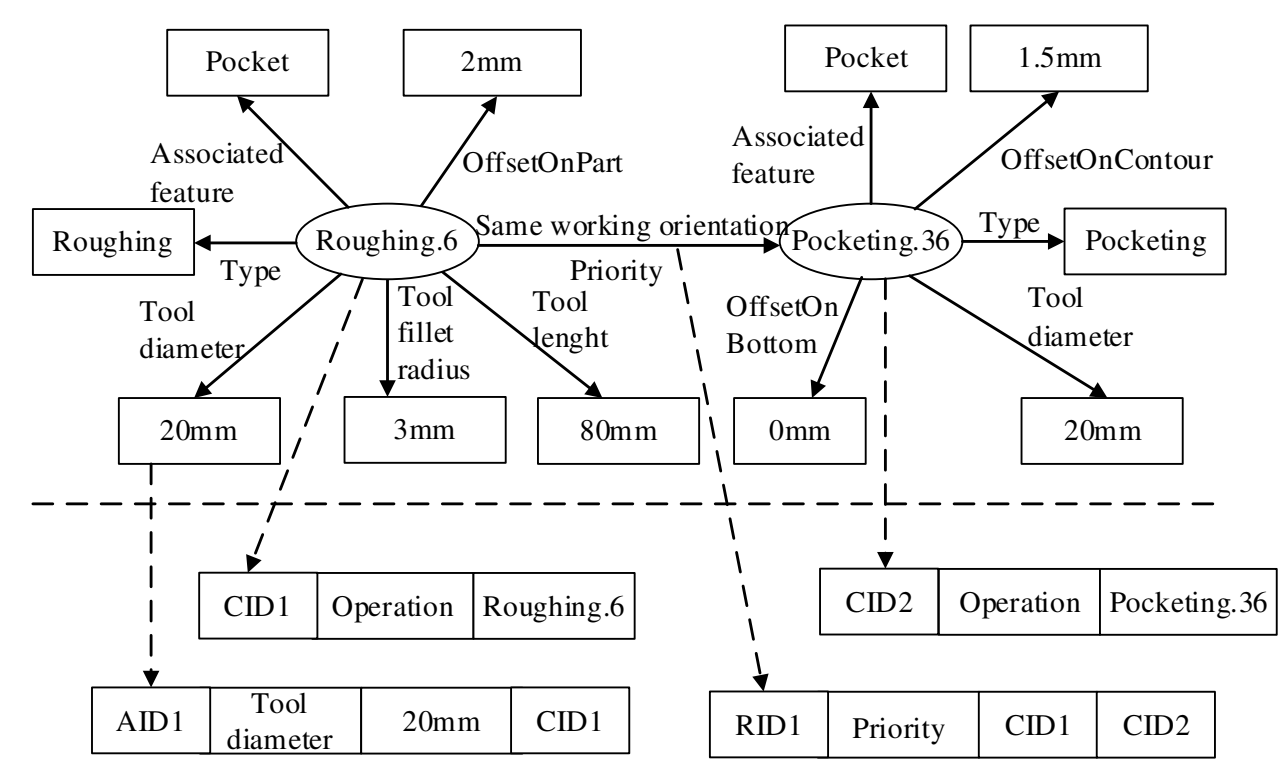

Fig. 3 Machining operation/working step semantic information description example

Through semantic description of machining operations or working steps and other information, the semantics and attributes of NC process information can be clearly described, thereby establishing a connection between low-level machining operation information and high-level process design intent.

\subsection{Similarity evaluation of process design intention}

After the process information is represented by the semantic graph, the similarity calculation of the semantic graph can be used to evaluate the similarity of the process design intent. Semantic graph similarity calculation includes concept similarity, concept relationship similarity and attribute similarity.

\section{(1) Concept similarity}

In concept matching, it is possible that the semantically matched concepts in the macro process and the micro process use different semantic descriptions, such as the concept of "machining allowance" in the macro process, and the concepts such as "bottom offset" and "profile offset" in the micro process all satisfy its semantics. Therefore, in addition to the direct semantic representation of the process information, the concept set needs to be semantically extended, and the similarity between the calculated concepts $C_{A i}$ and $C_{I j}$ is converted into the similarity between each element in the extended word set $C S_{A i}$ of the calculated concept $C_{A i}$ and $C_{I j}$, and then take the maximum value as the semantic similarity of $C_{A i}$ and $C_{I j}$, that is, for any two concepts $C_{A i}$ and $C_{I j}$ taken from $C_{A}$ and $C_{I}$ respectively, the similarity of the concept names is:

$$
\operatorname{Sim}_{n}\left(C_{A i}, C_{I j}\right)=\max _{C_{A i k} \in C S_{A i}}\left(2 C_{k} /\left(\left|C_{A i k}\right|+\left|C_{I j}\right|\right)\right)
$$

Where, $c_{k}$ is the number of characters contained in the common substring of two concepts.

Thus, the average name similarity between the concept set $C_{A}=\left\{C_{A 1}, C_{A 2}, \ldots, C_{A m}\right\}$ and $C_{I}=\left\{C_{I 1}, C_{I 2}, \ldots, C_{I n}\right\}$ in machining operations and working steps is: 


$$
\operatorname{Sim}_{n}\left(C_{A}, C_{I}\right)=\frac{1}{m} \sum_{i \in m} \max _{j \in n} \operatorname{Sim}_{n}\left(C_{A i}, C_{I j}\right)
$$

(2) Concept relationship similarity

The calculation of the previous formula only calculates the similarity of concepts without considering the relationship between these concepts. In fact, the relationship between concepts can well reflect the process context. For example, $W S_{a}$ and $W S_{b}$ are at the same working orientation, and $W S_{a}$ takes precedence over $W S_{b}$. Therefore, the conceptual relationship must be considered in the matching process.

Assuming that the concept relation set starting from the concept $C_{A i}$ is $R_{A i}=\left\{R_{A i 1}, R_{A i 2}, \ldots\right.$, $\left.R_{A i p}\right\}$, the concept relation set starting from the concept $C_{I j}$ is $R_{I j}=\left\{R_{I j 1}, R_{I j 2}, \ldots, R_{I j q}\right\}$, for any two association relations $R_{A i x}$ and $R_{I j y}$, the similarity calculation formula is:

$$
\operatorname{Sim}_{r}\left(R_{A i x}, R_{I j y}\right)= \begin{cases}1 & R_{A i x}=R_{l j y} \\ 0 & \text { others }\end{cases}
$$

Similarly, the relationship similarity between the concept $C_{A i}$ and the concept $C_{I j}$ is calculated as follows:

$$
\operatorname{Sim}_{r}\left(C_{A i}, C_{l j}\right)=\frac{1}{p} \sum_{x \in p} \sum_{y \in q} \operatorname{Sim}_{r}\left(R_{A i x}, R_{I j y}\right)
$$

Thus, the overall similarity of the relationship between machining operations and working steps is:

$$
\operatorname{Sim}_{r}\left(C_{A}, C_{I}\right)=\frac{1}{m} \sum_{i \in m} \sum_{j \in n} \operatorname{Sim}_{r}\left(C_{A i}, C_{l j}\right)
$$

(3) Attribute similarity

The attribute similarity is calculated by the following formula:

$$
\operatorname{Sim}\left(A_{A}, A_{I}\right)=\left\{\begin{array}{l}
\operatorname{Sim}_{n}\left(A_{A}, A_{I}\right) \text { type=int/double } \\
\operatorname{Sim}_{v}\left(A_{A}, A_{I}\right) \text { type=string }
\end{array}\right.
$$

Where, $\operatorname{Sim}_{n}\left(A_{A}, A_{I}\right)$ represents the name similarity between attribute $A_{A}$ and attribute $A_{I}, \operatorname{Sim}_{v}\left(A_{A}\right.$, $A_{I}$ ) represents the numerical similarity between attribute $A_{A}$ and attribute $A_{I}$, and type represents the data type of attribute.

Similar to the above concept name similarity calculation, the concept attribute name similarity is calculated by using the concept attribute set $A_{A i}=\left\{A_{A i 1}, A_{A i 2}, \ldots, A_{A i u}\right\}$ and $A_{I j}=\left\{A_{I_{j j} 1}\right.$, $\left.A_{I j 2}, \ldots, A_{I j v}\right\}$, specifically:

$$
\operatorname{Sim}_{n}\left(A_{A i}, A_{I j}\right)=\frac{1}{u} \sum_{s \in u} \max _{t \in v}\left(2 \mathrm{c} /\left(\left|A_{A i s}\right|+\left|A_{I j t}\right|\right)\right)
$$

Similarly, the overall attribute name similarity is:

$$
\operatorname{Sim}_{n}\left(A_{A}, A_{I}\right)=\frac{1}{m} \sum_{i \in m} \max _{j \in n} \operatorname{Sim}_{n}\left(A_{A i}, A_{I j}\right)
$$

The numerical similarity of attributes is calculated as follows:

$$
\operatorname{Sim}_{v}\left(A_{A}, A_{I}\right)=1-\frac{\left|v\left(A_{A}\right)-v\left(A_{I}\right)\right|}{\max \left(v\left(A_{A}\right), v\left(A_{I}\right)\right)}
$$

The similarity between working step $A$ of macro process and machining operation $I$ of micro process can be obtained by comprehensively weighting its conceptual similarity, conceptual relationship similarity and attribute similarity, expressed by $\delta$, then:

$$
\begin{aligned}
& \delta=\omega_{1} \operatorname{Sim}_{n}\left(C_{A}, C_{I}\right)+\omega_{2} \operatorname{Sim}_{r}\left(C_{A}, C_{I}\right)+\omega_{3} \operatorname{Sim}\left(A_{A}, A_{I}\right) \\
& \omega_{1}+\omega_{2}+\omega_{3}=1
\end{aligned}
$$




\subsection{Overall matching of macro process and micro process}

Consider each working step in the macro process and each machining operation in the micro process as a node, the process information describing the working step/machining operation as the attribute of the node, and the fusion between the macro process and the micro process can be seen as the optimal matching process of a complete bipartite graph composed of two nod sets.

Let $M_{1}$ and $M_{2}$ denote the fused micro process and macro process respectively, $u_{i}$ is the node of $M_{1}$, and $v_{j}$ is the node of $M_{2}$. The node $u_{i}$ in $M_{1}$ is paired with the node $v_{j}$ in $M_{2}$ in turn, the similarity $\delta_{i j}$ of each pair of nodes is calculated according to formula (11), and $\delta_{i j}$ is used as the weight of the bipartite graph, then the weight matrix of the bipartite graph is the fusion matrix $\boldsymbol{M}$ between $M_{1}$ and $M_{2}$, as shown in Fig. 4. When the number of nodes in the two groups is different, the matrix is supplemented with 0 to make the fusion matrix into a square matrix.

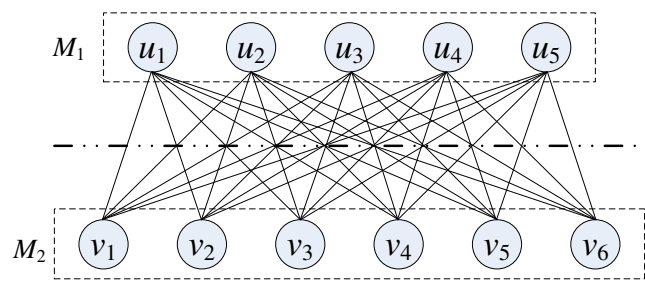

Fig. 4 Complete bipartite graph formed by process nodes

$$
\boldsymbol{M}=u_{3}\left[\begin{array}{cccccc}
u_{1} \\
u_{2} \\
u_{4} \\
u_{5} \\
0
\end{array}\left[\begin{array}{ccccccc}
\delta_{11} & \delta_{12} & \delta_{13} & \delta_{14} & \delta_{15} & \delta_{16} \\
\delta_{21} & \delta_{22} & \delta_{23} & \delta_{24} & \delta_{25} & \delta_{26} \\
\delta_{31} & \delta_{32} & \delta_{33} & \delta_{34} & \delta_{35} & \delta_{36} \\
\delta_{41} & \delta_{42} & \delta_{43} & \delta_{44} & \delta_{45} & \delta_{46} \\
\delta_{51} & \delta_{52} & \delta_{53} & \delta_{54} & \delta_{55} & \delta_{56} \\
0 & 0 & 0 & 0 & 0 & 0
\end{array}\right]\right.
$$

In order to obtain the best fusion result between the reusable macro processes and the reusable micro processes, the Kuhn-Munkres algorithm [26] which calculates the optimal matching of the weighted bipartite graph in graph theory is used to calculate the optimal matching scheme of the fusion matrix. The Kuhn-Munkres algorithm is a classic algorithm for solving the optimal matching of bipartite graphs in graph theory. This algorithm transforms the maximum full matching problem into constantly finding the augmented path by giving a label to each vertex, so that the matching of bipartite graph can achieve the optimal matching. Firstly, the definitions of optimal matching and augmented path are given.

Define 1 Optimal matching: for bipartite graph $G=(V(X, Y), E)$, if a matching $M$ can be found and satisfies the maximum weight sum of all matches, $M$ is called the optimal matching of $G$.

Define 2 Augmented path: if $M$ is a match of bipartite graph $G=(V(X, Y), E)$, let there be a path from one vertex to another in graph $G$. this path is composed of edges belonging to $M$ and edges not belonging to $M$ alternately, then this path is an augmented path. If the first and last vertices of the augmented path do not belong to $M$, the path is called an augmentable path.

The description of the Kuhn-Munkres algorithm is as follows:

Step1. Give the initial label $l\left(u_{i}\right)=\max _{j} \delta_{i j}, l\left(v_{j}\right)=0, i, j=1,2, \ldots, n$;

Step2. Find the edge set $E l=\left\{\left(u_{i}, v_{j}\right) \mid l\left(u_{i}\right)+l\left(v_{j}\right)=\delta_{i j}\right\}, G l=(V, E l)$ and one matching $M$ in $G L$ ( $M \subseteq E$ and any two edges in $M$ are not adjacent); 
Step3. If $M$ has saturated all vertices of $M_{1}$, then $M$ is the optimal matching of $G$, and the algorithm ends; otherwise, proceed to the next step;

Step4. Find an unsaturated vertex $x_{0}$ of $M$ in $M_{1}$, let $A \leftarrow\left\{x_{0}\right\}, B \leftarrow \emptyset, A$ and $B$ are two sets;

Step5. If $P_{G l}(A)=B$, turn to Step9, otherwise proceed to the next step, where $P_{G l}(A) \subseteq M_{2}$ is the set of vertices adjacent to the vertices in $A$;

Step6. Find one vertex $y \in P_{G l}(A)-B$;

Step7. If $y$ is the saturation vertex of $M$, find out the pairing vertex $z$ of $y$, make $A \leftarrow A \bigcup\{z\}$, $B \leftarrow B \cup\{y\}$, and turn to Step5; Otherwise, proceed to the next step;

Step8. There is an augmentable path $R$ from $x_{0}$ to $y$, make $M \leftarrow M \oplus E(R)$, turn to Step3;

Step9. Let $\alpha=\min \left\{l\left(u_{i}\right)+l\left(v_{j}\right)-\delta_{i j}\right\}$, where $u_{i} \in A, v_{j} \notin P_{G I}(A)$; Modify the label as shown in equation (13), and calculate $E l^{\prime}$ and $G l^{\prime}$ according to $l$ ';

$$
l^{\prime}(v)= \begin{cases}l(v)-\alpha & v \in A \\ l(v)+\alpha & v \in B \\ l(v) & \text { others }\end{cases}
$$

Step10. $l \leftarrow l ', G l \leftarrow G l$, turn to Step6.

\subsection{Compatibility credibility evaluation model construction}

According to the above optimal matching scheme, the similarity $S$ of the fusion of a macro process and a micro process is defined as the sum of the similarity of the optimal matching process nodes. The specific calculation formula is as follows

$$
S=\frac{1}{n} \sum_{j=1}^{n} \delta_{m(j) j}
$$

Where, $\delta_{m(j) j}$ represents the similarity between the $m(j)$-th working step of macro process $M_{1}$ and the $j$-th machining operation of micro process $M_{2}, m(j)$ represents the number of rows optimally matched with the $j$-th column in equation (12), and $n$ is the number of matching nodes.

The NC process scheme of the query part is quickly generated by macro process driven similar NC process fusion of multi-source local structure, that is, it is obtained by the fusion of one macro process and multiple micro processes. In this way, the compatibility credibility evaluation model of fusion results can be constructed by the similarity of fusion results between multiple micro processes and one macro process. In addition, the more nodes included in the micro process, the greater the impact on the similarity of fusion results. Therefore, considering the influence of nodes contained in different similar NC processes on the similarity of fusion results, the specific calculation formula of compatibility credibility evaluation model $C$ for the NC process scheme obtained by the fusion of the query part is as follows

$$
C=\sum_{k=1}^{m}\left(n_{k} S_{k}\right) / \sum n_{k}
$$

Where, $S_{k}$ represents the similarity between the fusion results of the macro process and the $k$-th micro process of local structure, and $n_{k}$ represents the number of machining operations contained in the $k$-th micro process of local structure.

\section{Optimization and adjustment of machining area}

According to the calculation results of compatibility credibility evaluation model, the fusion results of macro process and micro process are divided into credible and incredible. When it is not credible, it indicates that the micro process is incompatible with the macro process. It is necessary to reselect the macro/micro process and iterate the evaluation again until a compatible process scheme is obtained. When the fusion result is credible, the machining area corresponding to the 
process scheme needs to be optimized and adjusted from the geometric level.

The optimization and adjustment of machining area mainly realizes the integration of machining areas and the optimization of machining sequence. Due to the interaction between machining features, an efficient process scheme cannot be obtained only through the fusion of process semantics in the previous section. Therefore, under the guidance of macro process, it is necessary to integrate the machining areas according to the principle of making full use of cutting tool machining ability, and realize "one size fits all" as far as possible. This paper intends to analyze the machining areas that may participate in the fusion by constructing the interaction relationship between features and combined with the machining areas calculation of working step, and use swarm intelligence algorithm to optimize the machining sequence of the fused machining areas.

\subsection{Interaction between features and calculation of machining area}

The constraint relationship between machining areas is one of the important factors to be considered in the process of machining area fusion. According to the needs of machining region fusion, this paper establishes the constraint relationship between machining areas based on the interaction between features. Machining feature interaction can be divided into two types: design-coupled relation $\left(R_{D}\right)$ and manufacturing-coupled relation $\left(R_{M}\right)$, as shown in Fig. 5. The former mainly associates manufacturing features through tolerance annotation, and the latter mainly includes dependent relation and adjacent relation, which can reflect the machining sequence among machining features.

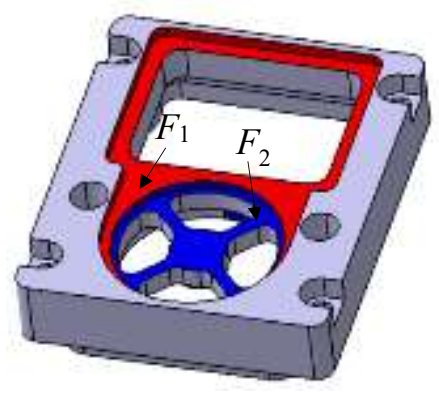

a Dependent relation

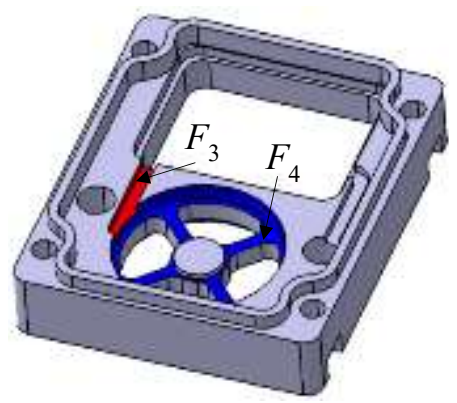

b Adjacent relation

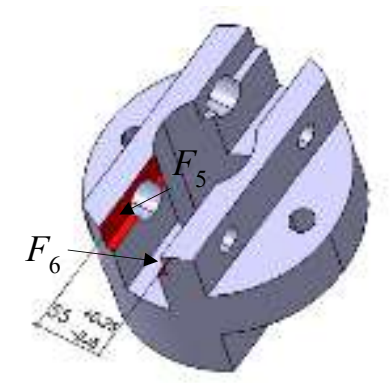

c Design-coupled relation

Fig. 5 Machining feature interaction

The transformation of the above-mentioned interaction relationship between features into the constraint relationship between machining areas needs to be established on the premise of the same tool axis direction, and is also related to the process intent, that is, different working steps show different machining areas, such as roughing and re-roughing, which are related to the cutting volume, while the profile finishing usually only considers the profile surface due to the small amount of radial machining. These calculations are related to the machining area.

The medial axis (MA) of the pocket is defined as the center track that can cover the whole region moving along the pocket contour. This track geometrically divides the pocket area and can represent the largest feasible cutting tool. Therefore, this paper uses the method of medial axis transformation to calculate the machining area. Firstly, the original contour is obtained by offsetting the contour of the area to be machined according to the allowance; Then, the medial axis of the pocket is calculated, and the supplementary contour is obtained by making an arc based on the vertex of the medial axis; Finally, the machining area of the cutting tool is constructed by combining the original contour and the supplementary contour. Specifically, the method proposed 
in reference [27] is adopted.

Fig. 6 shows an example of calculating the machining area of a pocket. In Fig. 6a, the area $C_{3}$ needs to be processed, the white line segment is its contour line $L_{1}$, Fig. $6 \mathrm{~b}$ shows the medial axis MA calculated by the cutting tool $R$ and its corresponding supplementary contour line $L_{2}$, and then the machining areas $C_{1}$ and $C_{2}$ corresponding to cutting tool $R$ are calculated in combination with $L_{1}$ and $L_{2}$, as shown in Fig. 6c.

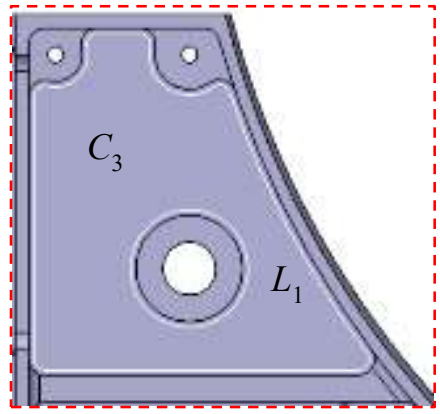

a Contour

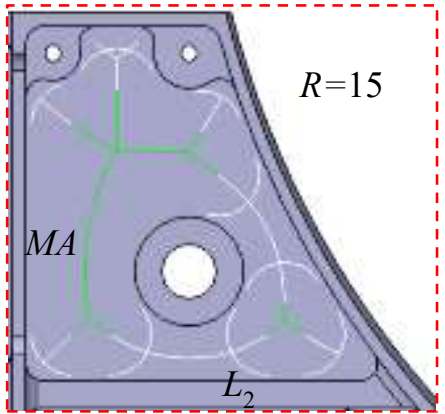

b Medial axis

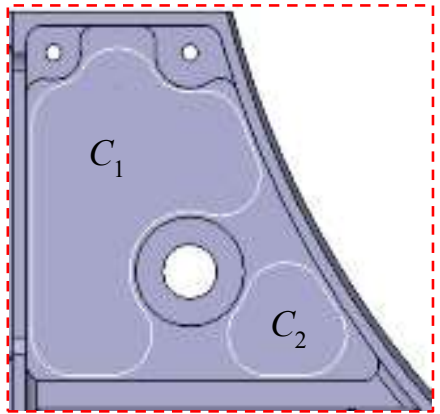

c Machining area

Fig. 6 Machining area calculation based on medial axis transformation

\subsection{Machining area fusion analysis based on design structure matrix}

In the process of $\mathrm{NC}$ process design based on machining features, the machining areas under different working steps are attached to machining features, which are related to the process information such as working procedure and working step. After planning the macro process, the information granularity of machining features is too coarse for the process planning at the micro level. Therefore, in the process planning of micro layer, the relationship between machining areas needs to be considered. In the current process planning system, this analysis is usually obtained by experienced process designer. However, when the part structure is complex, the number of machining areas involved in the process is large, and the process designer cannot accurately obtain the constraint relationship between machining elements, that is, they cannot make effective judgment on cutting tool adoption and machining sequence. Therefore, in this paper, the design structure matrix (DSM) is used to analyze the machining area, and the machining area in the process is divided into different modules according to the constraint relationship. Each module is as independent as possible from other modules, that is, the machining areas between modules do not affect each other. When one cutting tool is used for machining, the same module shall be processed together as much as possible. Therefore, the process designer only needs to consider the machining sequence of the machining areas between different modules, so as to simplify the workload and improve the optimization efficiency and quality.

Design structure matrix (DSM) [28] is a matrix representation method of object dependency proposed by Steward. Based on the existing algorithms and according to the constraint relationship of machining area, this paper proposes a machining area fusion analysis method based on design structure matrix.

Assuming that there are $n$ machining areas in the part process scheme fused according to the method in the previous section, the machining area sequence $M R_{i}=(i=1,2, \ldots, n)$ is constructed according to the sequence in the process scheme, and the method shown in Fig. 7 is used to construct $n \times n$-order square matrix $\boldsymbol{A}$. In the $n \times n$-order square matrix $\boldsymbol{A}$, the main diagonal elements represent the machining region itself, and the non-main diagonal elements represent the constraint relationship with other machining regions. If the machining area $M R_{i}$ is processed 
before the machining area $M R_{j}$, the element $a_{i j}=1$, otherwise $a_{i j}=0$, where $i \neq j$.

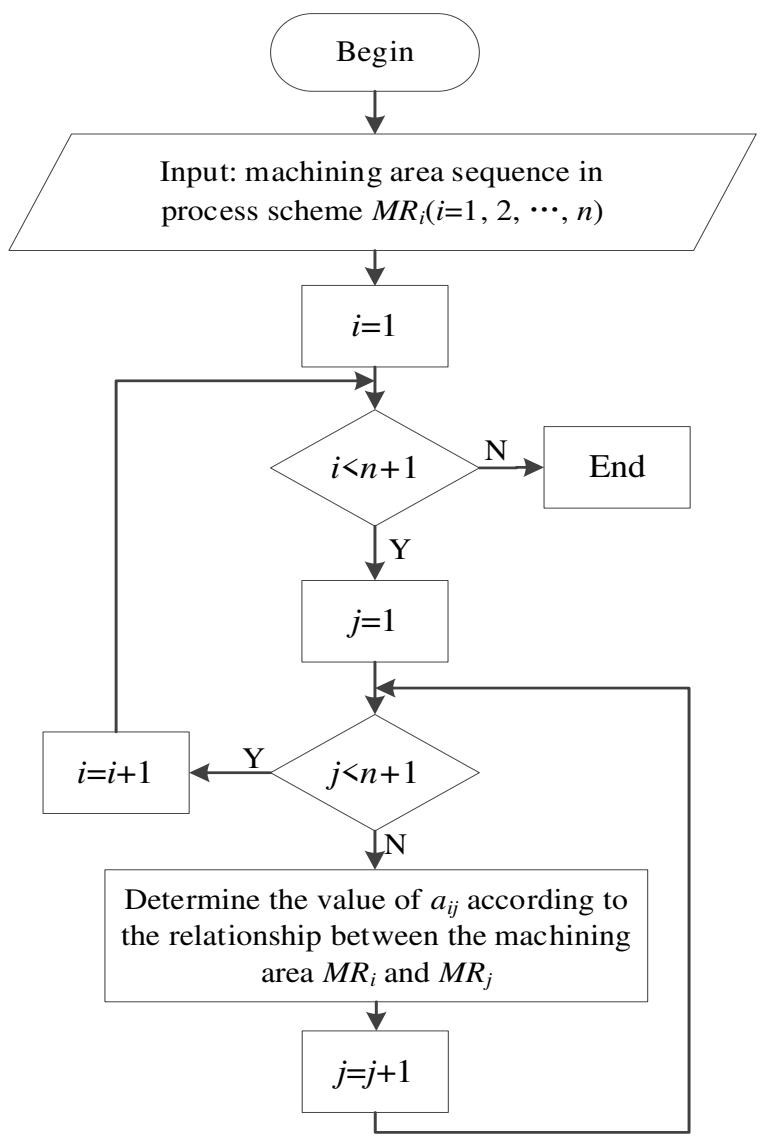

Fig. 7 DSM matrix construction flow chart

Fig. 8 shows the initial DSM matrix constructed with 10 machining areas. The elements below the diagonal line indicate the positive relationship, and the elements above the diagonal line indicate the reverse relationship. If there are elements above and below the diagonal, it indicates that there is an association relationship between machining areas, but there is no machining sequence relationship.

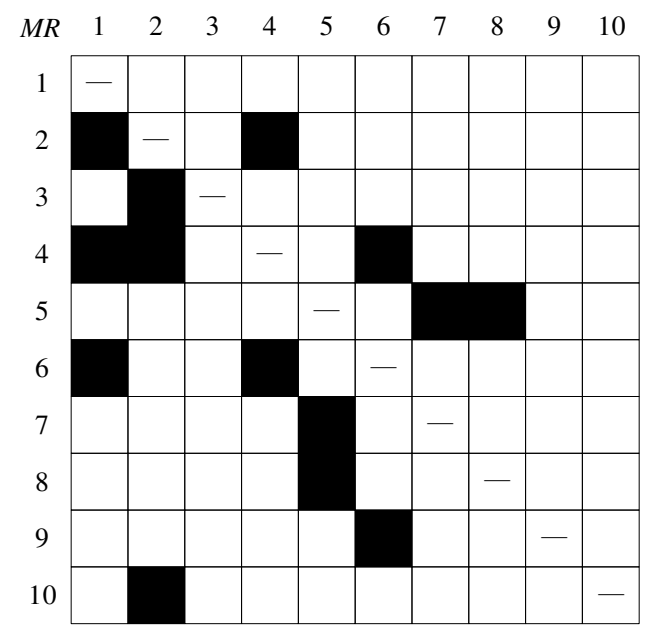

Fig. 8 Initial DSM matrix

After the initial matrix construction is completed, the machining area clustering and fusion analysis are required. This paper uses the algorithm in Table 1 to obtain the clustering matrix $\boldsymbol{M}$, 
and conducts the fusion analysis based on the matrix $\boldsymbol{M}$.

Table. 1 DSM clustering algorithm

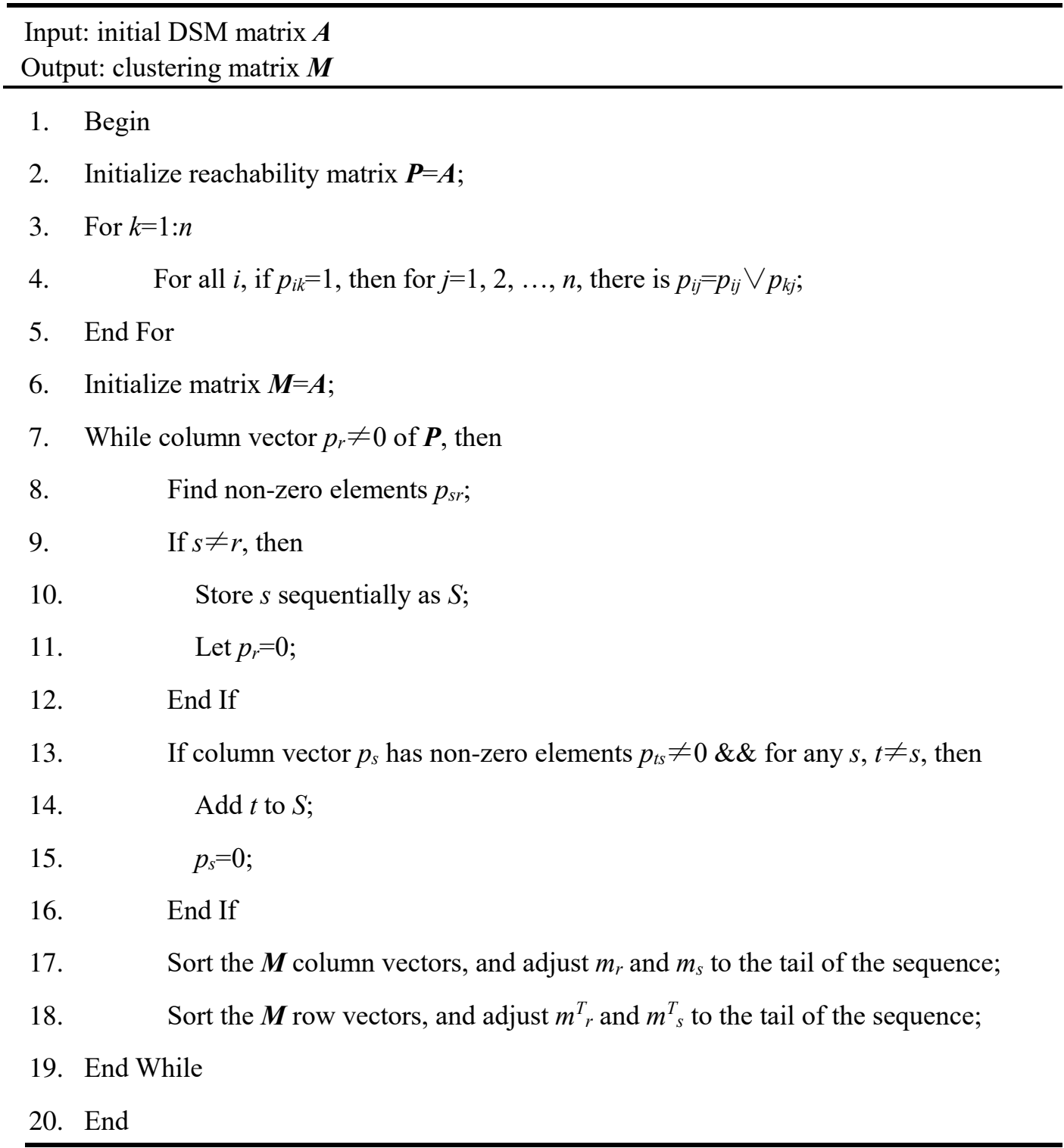

If $P_{i j}=1$ in the reachable matrix $\boldsymbol{P}$, it means that there is a non-zero length path from element $i$ to element $j$. If the $j$-th column of the matrix $\boldsymbol{P}$ is a non-zero element at the $i_{1}$-th, $i_{2}$-th, $\ldots, i_{l}$-th row, then the element $j$ to the elements $i_{1}, i_{2}, \ldots, i_{l}$ are all reachable, that is, in the process of using the same cutting tool, the machining of the element $j$ can pass through at most $l$ steps to the machining of element $i$. The clustering matrix is obtained through the algorithm in Table 1, as shown in Fig. 9 . 


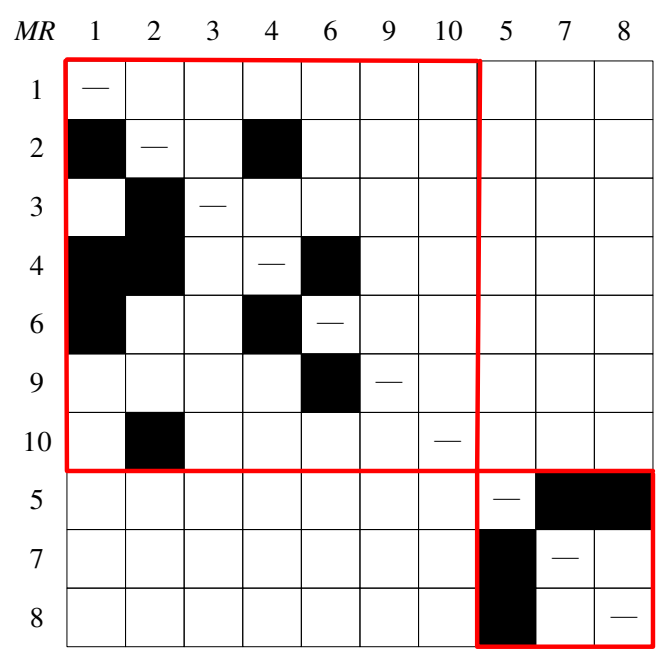

Fig. 9 DSM clustering matrix

It can be seen from the above that after clustering, the machining areas in the process can be divided into several sub-blocks. The machining areas in the same sub-block have a constraint relationship, and can be processed by the same cutting tool at one time. The machining areas in different sub-blocks do not have a constraint relationship, that is, processing can be arranged separately. Therefore, in the subsequent process optimization, the process designer only needs to consider the machining sequence between the sub-blocks, which greatly simplifies the complexity of the modification.

\section{Machining sequence optimization based on swarm intelligence}

After the fusion of the $\mathrm{NC}$ processes is completed, the machining sequence of the machining areas under each working step is less related to the process scheme, so the swarm intelligence algorithm can be used to optimize the geometric path between the machining areas. At the same time, through the aforementioned analysis, the machining priority relationship and the internal constraint relationship can be obtained, which can be used as the initial value to effectively reduce the complexity of the optimization process.

\subsection{The machining sequence representation and optimization goal}

Given a machining area group of a working step $S=\left\{c_{1}, c_{2}, \ldots, c_{n}\right\}, S$ is a set of machining areas belonging to the working step in all feature NC process chains of a part. After sorting the machining areas in a certain order, a group of machining area sequences $q\left(c_{1}{ }^{\prime}, c_{2}{ }^{\prime}, \ldots, c_{n}{ }^{\prime}\right), c_{i}{ }^{\prime} \in S$, $1 \leq i \leq n$ is obtained. The sequence represents a machining path between machining areas. The positions between machining areas can be arranged randomly. In theory, there are $n$ ! kinds of machining area sequences, and the machining areas need to meet the constraint relationship, so the actual number of machining area sequences should be less than $n$ !. Since each machining area has its own code, in order to simplify the problem description, the path sequencing problem of the machining area is transformed into $n$ positive integer sequencing problems. In this way, $q(1,2, \ldots$, $n$ ) can be used to represent $q\left(c_{1}{ }^{\prime}, c_{2}{ }^{\prime}, \ldots, c_{n}{ }^{\prime}\right)$. The solution space of machining area sequence is expressed as: $D=\left\{\left(i_{1}, i_{2}, \ldots, i_{n}\right) \mid i_{j}\right.$ represents the $j$-th element number of machining operation sequence $\left(c_{1}{ }^{\prime}, c_{2}{ }^{\prime}, \ldots, c_{n}{ }^{\prime}\right)$, and $\left.i_{j} \neq i_{m}, j \neq m, 1 \leq j \leq n, 1 \leq m \leq n\right\}$.

Set the initial solution as $(1,2, \ldots, n)$, representing the initial machining region sequence as $c_{1}, c_{2}, \ldots, c_{n}$.

Given a machining area group, a set of machining area sequence is solved under the 
condition of meeting machining constraints, and the machining path of the machining area sequence is the shortest. Its mathematical description model is:

$$
g(S)=\min \sum_{j=1}^{n} d\left(c_{i j}, c_{i(j+1)}\right)
$$

Where $d\left(c_{i j}, c_{i(j+1)}\right)$ represents the distance between the machining operation $c_{i j}$ and $c_{i(j+1)}$.

\subsection{Acceptance criteria}

In order to evaluate the pros and cons of the new solution generated in the $i$-th path optimization iteration compared with the initial solution, the cost difference between the new solution and the initial solution is calculated by the following formula:

$$
\Delta g_{i}=g\left(S_{i}^{\prime}\right)-g\left(S_{i}\right)
$$

Where, $S_{i}^{\prime}$ and $S_{i}$ are the new solution and initial solution produced by the $i$-th iteration respectively, and its acceptance function is constructed using Metropolis probability acceptance criteria.

$$
S_{i+1}=\left\{\begin{array}{l}
S_{i}^{\prime}, \Delta g_{i}<0 \| \exp \left(-\Delta g_{i} / t_{k}\right)>\operatorname{random}(0,1) \\
S_{i}, \Delta g_{i}=0 \|\left(\Delta g_{i}>0 \& \& \exp \left(-\Delta g_{i} / t_{k}\right) \leq \operatorname{random}(0,1)\right)
\end{array}\right.
$$

Where, $t_{k}$ is the annealing temperature, and $\operatorname{random}(0,1)$ can produce decimals in the interval $[0,1]$.

\subsection{Machining area sequence optimization based on chaos-simulated annealing}

The inherent ability of the simulated annealing (SA) algorithm to avoid falling into local optimization makes it one of the most prominent algorithms for solving complex nonlinear problems. In traditional simulated annealing (SA), the most important step is the generation of random initial solutions. Most studies have shown that random sequences can affect the convergence speed of the simulated annealing algorithm. Chaos is a kind of motion similar to random motion. It can traverse all possible states according to its own laws without repeating it. It has the characteristics of randomness, ergodicity and regularity. It uses chaotic variables for optimized search and has good global search capabilities. By introducing chaotic variables into the simulated annealing algorithm and using the chaotic system to construct a chaotic sequence, the problem to be solved is converted to the chaotic space, which can improve the convergence speed and global search performance of the simulated annealing algorithm. Therefore, this paper introduces chaotic variables into the simulated annealing algorithm and replaces Gaussian distribution with chaotic random sequence numbers to expand the random search space.

In this paper, the chaotic random sequence function is generated by using Logistic mapping, in which the Logistic equation is a simple nonlinear parabolic equation, expressed as:

$$
x_{k+1}=\lambda x_{k}\left(1-x_{k}\right), \quad k=0,1,2 \ldots
$$

Where, $\lambda$ represents the control parameter and $\lambda \in(0,4]$, when $3.57<\lambda \leq 4$, the random sequence generated by Logistic equation is in a chaotic state, $\lambda=4$ is used to ensure that the random sequence generated by the Logistic equation is in a completely chaotic state in this paper.

The chaotic simulated annealing algorithm is described in detail below, and the steps are as follows: 
Step1. Initialize cooling progress parameters (including initial cooling temperature $t_{s}$, termination temperature $t_{e}$, Mapkov chain length $L_{k}$ and attenuation coefficient $\sigma$ ), and generate random initialization machining operation sequence $q_{0}$;

Step2. Under the control of the current cooling temperature $t_{s}$ and the number of iterations $i=1,2, \ldots, L_{k}$, repeat Step3 $\sim$ Step5 for each cooling temperature $t_{s}$;

Step3. For the initial machining operation sequence $q_{i}$ under the current iteration, a new machining operation sequence $q_{i}^{\prime}$ is generated by referring to the chaotic random sequence generation function;

Step4. Calculate the functional cost difference $\Delta g_{i}$ between the new machining operation sequence $q_{i}^{\prime}$ and the initial machining operation sequence $q_{i}$ according to formula (17);

Step5. According to formula (18), judge whether to accept the new machining operation sequence $q_{i}^{\prime}$, if accepted, then $q_{i+1}=q_{i}^{\prime}$; otherwise, $q_{i+1}=q_{i}$;

Step6. After $L_{k}$ iterations, use the attenuation function to perform annealing to cool down;

Step7. Judge whether the iterative termination condition is satisfied, if it is satisfied, the current solution is the optimal solution, the optimal solution is output, and the program ends; otherwise, it returns to Step2 and continues the iterative calculation. When there is no change in the length of several adjacent Mapkov chains, the iteration is exited.

In the chaotic simulated annealing algorithm, the cooling schedule is a set of parameters used to control the process. A reasonable selection of the cooling schedule parameters can improve the performance of the algorithm and reduce the CPU running time. Its parameters mainly include: initial temperature $t_{s}$, attenuation function, Mapkov chain length $L_{k}$ and stopping criteria.

1. Initial temperature

In the traditional simulated annealing algorithm, in order to make the initial random search very sufficient, a higher initial temperature is usually selected, but this actually causes a lot of redundant iterations. First, assume the probability of accepting the deteriorating solution is $p_{r 0}$, according to the Metropolis probability acceptance criterion $p_{r 0}=\exp \left(-\Delta g_{i} / t_{k}\right)$, so it can be deduced that $t_{k}=-\Delta g_{i} / \ln p_{r 0}$, and since the greater the acceptance probability of the deteriorating solution, the easier it is to reach the quasi-equilibrium, $p_{r 0}=0.8$ is set in this paper.

\section{Attenuation function}

Selecting a smaller temperature attenuation coefficient can ensure the quality of the final solution, but it will increase the CPU running time. Therefore, it is necessary to reasonably select the temperature attenuation coefficient. The attenuation function is expressed as:

$$
t_{k+1}=\sigma t_{k}, k=0,1,2, \ldots
$$

Where, $t_{\mathrm{k}+1}$ and $t_{\mathrm{k}}$ respectively represent the $k+1$-th iteration temperature and the $k$-th iteration temperature, and $\sigma$ represents the attenuation coefficient, and the selection range of $\sigma$ is usually $0.6 \sim 0.95$. In order to not only ensure the quality of the final solution but also reduce the CPU running time, this paper chooses $\sigma=0.8$.

\section{Mapkov chain length $L_{k}$}

Since there is an exponential growth relationship between the solution scale $n$ and the solution space, the selection of the Mapkov chain length $L_{k}$ is related to the solution scale $n$. In this paper, $L_{k}=50 n$ is selected.

\section{Stopping criteria}

The stopping criterion refers to the rule used to control the final stop of the algorithm. If there 
is no change in the length of several adjacent Mapkov chains, the algorithm terminates. Under reasonable initial temperature and Mapkov chain length parameters, this method can guarantee both the quality of the optimal solution and the efficiency of CPU operation, but it is not suitable for large-scale combinatorial optimization problems. The number of machining operations included in each working step of conventional parts is in the range of tens to hundreds, and the scale is not large. Therefore, when the solution does not change in the length of $m=1$ successive Mapkov chain, the algorithm is terminated, and $m$ means the number of times that the solution has no change in the length of the Mapkov chain.

\section{Case study}

To verify the feasibility and effectiveness of our approach presented in this paper, the similar $\mathrm{NC}$ process fusion algorithm of multi-source local structure is implemented on the platforms of Microsoft Visual Studio 2008 and CATIA V5 R21 component application architecture (CAA). The test is executed on a PC with Intel Core i3 CPU $3.40 \mathrm{GHz}$ and $4 \mathrm{~GB}$ memories.

\subsection{Compatibility evaluation case}

Fig. 10 shows a query plate part $C P$, a reusable macro process and a reusable micro process from the search results are selected, and the effectiveness of the algorithm is verified through the fusion process and results between them. Table 2 shows the reusable macro process information of similar part $S P$, including 5 working steps of Rough Milling Pocket, Semi-finish Milling Pocket Contour, and Finish Milling Pocket. Table 3 and Table 4 are the reusable micro process information of similar feature $S F$, including 4 machining operations such as Pocketing and Contouring.

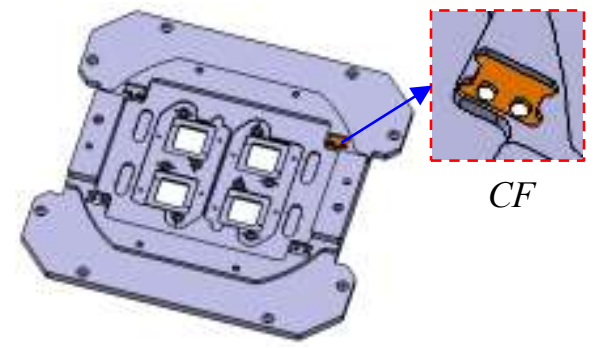

a Query part $C P$

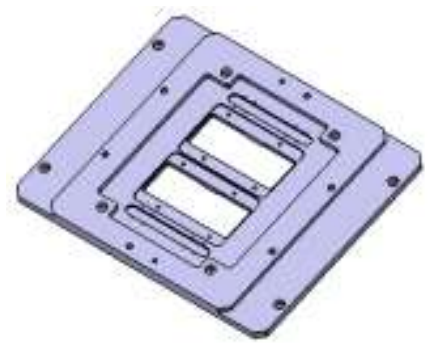

b Similar part $S P$

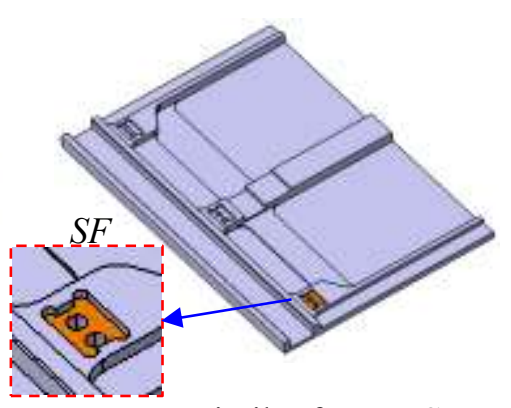

c Similar feature $S F$

Fig. 10 Example of two integrated processes

Table. 2 Reusable macro process information for similar part $S P$

\begin{tabular}{|c|c|c|c|c|c|c|c|c|c|c|c|c|c|c|c|}
\hline & \multicolumn{3}{|c|}{$W S_{1}$} & \multicolumn{3}{|c|}{$W S_{2}$} & \multicolumn{3}{|c|}{$W S_{3}$} & \multicolumn{3}{|c|}{$W S_{4}$} & \multicolumn{3}{|c|}{$W S_{5}$} \\
\hline Type & \multicolumn{3}{|c|}{$\begin{array}{c}\text { Rough Milling } \\
\text { Pocket }\end{array}$} & \multicolumn{3}{|c|}{$\begin{array}{l}\text { Rough Milling } \\
\text { Pocket }\end{array}$} & \multicolumn{3}{|c|}{$\begin{array}{c}\text { Semi-finish } \\
\text { Milling Pocket } \\
\text { Contour }\end{array}$} & \multicolumn{3}{|c|}{$\begin{array}{c}\text { Finish Milling } \\
\text { Pocket }\end{array}$} & \multicolumn{3}{|c|}{$\begin{array}{c}\text { Finish Milling } \\
\text { Pocket }\end{array}$} \\
\hline \multirow{2}{*}{$\begin{array}{l}\text { Cutting } \\
\text { tool }\end{array}$} & $D$ & $T_{L}$ & $r$ & $D$ & $T_{L}$ & $r$ & $D$ & $T_{L}$ & $r$ & $D$ & $T_{L}$ & $r$ & $D$ & $T_{L}$ & $r$ \\
\hline & 32 & 200 & 3 & 10 & 50 & 1.5 & 20 & 90 & 2 & 20 & 90 & 0 & 8 & 40 & 0 \\
\hline \multirow{2}{*}{$\begin{array}{l}\text { Mahcining } \\
\text { allowance }\end{array}$} & $\delta_{a}$ & & $\delta_{r}$ & $\delta_{a}$ & & $\delta_{r}$ & $\delta_{a}$ & & & $\delta_{a}$ & & $\delta_{r}$ & $\delta_{a}$ & & $r$ \\
\hline & 0.5 & & 1 & 0.5 & & 0.5 & 0.5 & & & 0 & & 0 & 0 & & 0 \\
\hline
\end{tabular}

Table. 3 Reusable micro process information of similar feature $S F$ (machining strategies)

\begin{tabular}{lllllll}
\hline op & Type & Cutting & Bottom & Contour & Cutting parameters & ... Cutting \\
\hline
\end{tabular}




\begin{tabular}{lcccccccccc}
\hline & & mode & offset & offset & $\begin{array}{c}v_{c} \\
\mathrm{~mm} / \mathrm{mn}\end{array}$ & $\begin{array}{c}f \\
\mathrm{~mm} / \mathrm{mn}\end{array}$ & $\begin{array}{c}a_{e} \\
\mathrm{~mm}\end{array}$ & $\begin{array}{c}a_{p} \\
\mathrm{~mm}\end{array}$ & & tool $T$ \\
\hline$o p_{1}$ & Pocketing & Helical & 0.3 & 0.3 & 300 & 1000 & 5 & 1.5 & $\ldots$ & 1 \\
$o p_{2}$ & Pocketing & Helical & 0.3 & 0.3 & 300 & 950 & 1.5 & 1 & $\ldots$ & 3 \\
$o p_{3}$ & Pocketing & Helical & 0 & 0.3 & 500 & 950 & 4 & 0.5 & $\ldots$ & 2 \\
$o p_{4}$ & Contouring & Parallel & 0 & 0 & 500 & 800 & 1.5 & 1 & & 3 \\
\hline
\end{tabular}

Table. 4 Reusable micro process information of similar feature $S F$ (cutting tool parameters)

\begin{tabular}{ccccccc}
\hline $\begin{array}{c}\text { Cutting } \\
\text { tool } T\end{array}$ & Type & Diameter/mm & Length $/ \mathrm{mm}$ & $\begin{array}{c}\text { Cutting } \\
\text { length } / \mathrm{mm}\end{array}$ & $\begin{array}{c}\text { Corner } \\
\text { radius } / \mathrm{mm}\end{array}$ & $\cdots$ \\
\hline 1 & End Mill & 10 & 100 & 55 & 2 & $\ldots$ \\
2 & End Mill & 8 & 100 & 40 & 0 & $\ldots$ \\
3 & End Mill & 3 & 30 & 15 & 0 & $\ldots$ \\
\hline
\end{tabular}

Table. 5 Comparison of node similarity between macro process and micro process

\begin{tabular}{cccccc}
\hline & $W S_{1}$ & $W S_{2}$ & $W S_{3}$ & $W S_{4}$ & $W S_{5}$ \\
\cline { 1 - 3 } \cline { 5 - 6 }$p_{1}$ & 0.387 & 0.858 & 0 & 0.346 & 0.494 \\
$o p_{2}$ & 0.145 & 0.312 & 0 & 0.260 & 0.399 \\
$o p_{3}$ & 0.135 & 0.572 & 0 & 0.446 & 0.900 \\
$o p_{4}$ & 0.055 & 0.192 & 0.100 & 0.460 & 0.599 \\
\hline
\end{tabular}

The similarity value of macro process and micro process fusion is calculated, as shown in Table 5, and the fusion matrix $\boldsymbol{M}$ is as follows

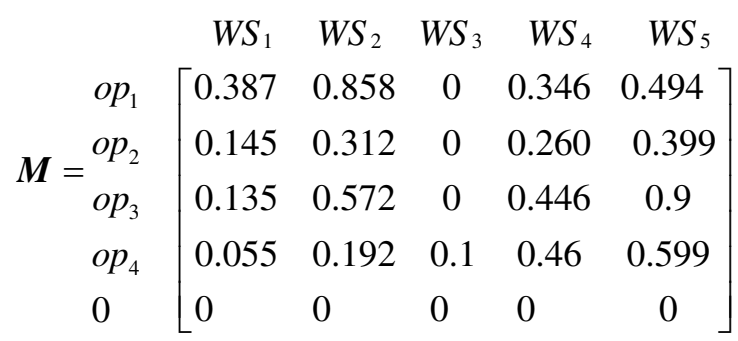

Using Kuhn-Munkres algorithm, an optimal matching solution $M$ between macro process and micro process is obtained, and then the matching with low similarity value is removed. Finally, $M=\left\{\left(o p_{1}, W S_{2}\right),\left(o p_{3}, W S_{5}\right)\right\}$ is determined. According to the optimal matching weight, the similarity value $S=0.620$ of the fusion of the two processes is calculated by formula (14), and it can be judged that the two processes are compatible. In addition, it can be seen that $o p_{2}$ and $o p_{4}$ are not matched by the macro process, so it is necessary to adjust the macro process and add the additional working steps. In this example, the working steps of D10 and D3 cutting tools are added for supplementary machining.

\subsection{Machining area fusion analysis case}

Fig. 12 shows an example of machining area fusion of parts Part1 and Part2 in Fig. 11. The part Part1 contains 15 sub-machining areas SMR in a certain working step Roughing, as shown in Fig. 11a. Through the analysis of the interaction relationship between features, the DSM matrix is constructed as shown in Fig. 12a. It can be seen that 15 sub-machining areas can be combined into one machining area $M R$ to support more efficient machining. Compared with Part1, Part2 has 22 sub-machining areas in the roughing stage. Through DSM clustering, 6 new machining areas $\left(S M R_{1}+S M R_{2} \rightarrow M R_{1}, S M R_{4}+S M R_{5} \rightarrow M R_{2}, S M R_{7}+S M R_{8} \rightarrow M R_{3}, S M R_{12}+S M R_{11}+S M R_{10}+S M R_{9}\right.$ $\rightarrow M R_{4}, S M R_{14}+S M R_{15}+S M R_{16}+S M R_{17}+S M R_{18}+S M R_{19} \rightarrow M R_{5}, S M R_{20}+S M R_{21} \rightarrow M R_{6}$ ). In addition, 
the three sub-machining areas $S M R_{3}, S M R_{6}$, and $S M R_{22}$ are independent. In the same way, it is also possible to analyze other machining stages, such as Finish Milling Inner Shape. Thus, the sub-machining area of each stage under the macro process can be fused and analyzed, and the machining area can be dynamically combined according to the process intention and manufacturing resources.

The combined sub-machining areas can inherit the interaction relationship of features, and its machining method (such as depth-first or layered machining) is related to the geometric characteristics of the sub-machining area.

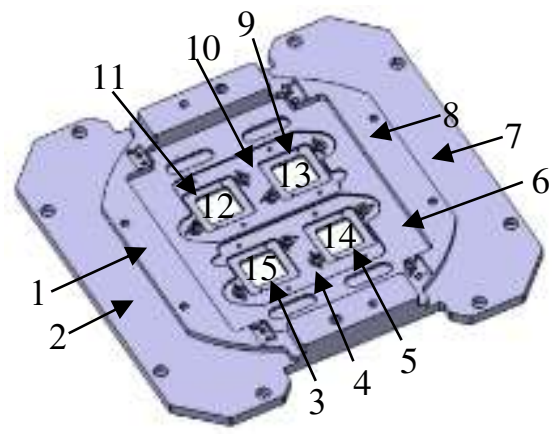

a Machining areas of Part1

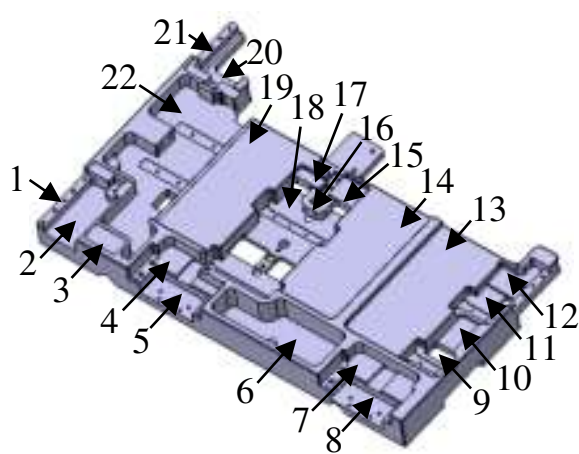

b Machining areas of Part2

Fig. 11 Part examples

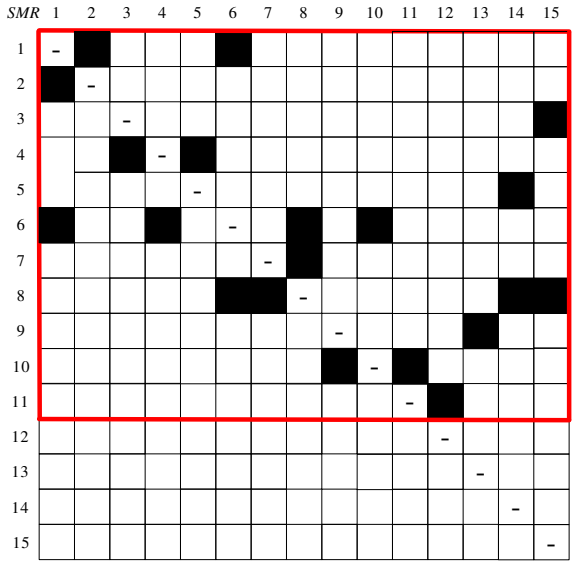

a DSM matrix of Part1

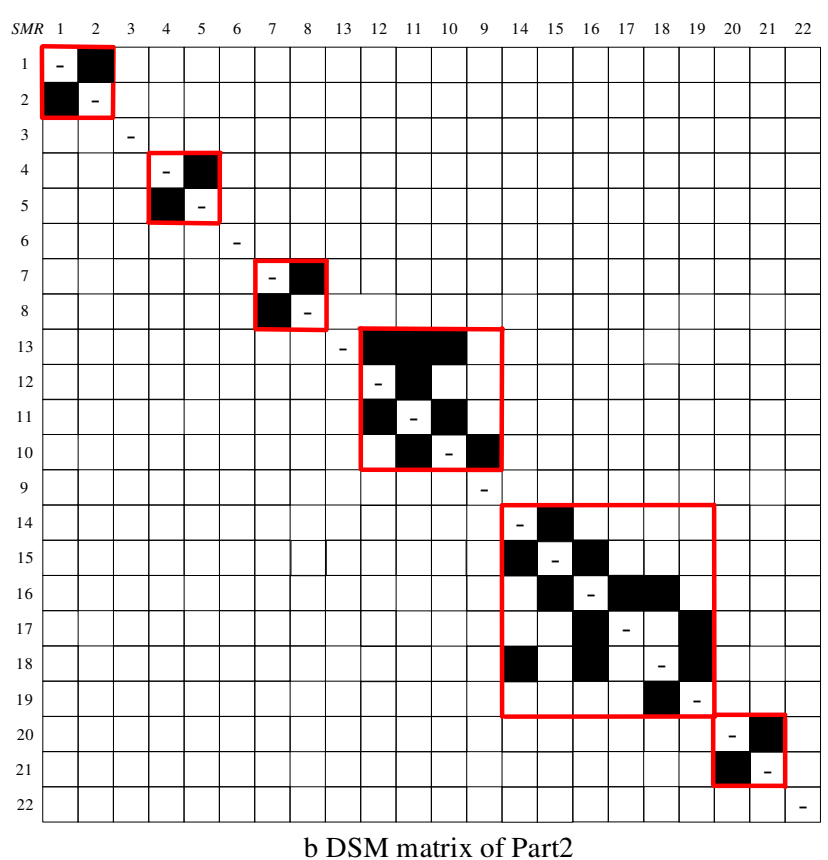

b DSM matrix of Part2

Fig. 12 DSM clustering of machining areas

\subsection{Machining sequence optimization case}

Fig. 13 shows a more complex part example Part3 to describe the feasibility and effectiveness of the machining sequence optimization method. Part3 is an aircraft structural part, which is mainly based on pocket machining. Among them, there will be sub-features in the pocket features, such as sags and bosses, which are processed in the form of multi-layer pockets after the machining areas are merged. In addition, the macro process is relatively standardized, mainly 
using the working steps of Roughing, Finish Milling Bottom, Finish Milling Corner, and Finish Milling Contour. Therefore, the machining sequence has a greater impact on its process scheme. This paper conducts optimization tests on the machining area sequence in three of the working steps. Fig. 13b shows the optimized tool path (red line) of the machining areas under Roughing, which contains 69 machining areas. The pink line in Fig. 13c is the optimized result of the machining areas under the Finish Milling Bottom. After Roughing and Finish Milling Bottom, the residual material at the 263 corners is processed, as shown in the green line in Fig. $13 \mathrm{~d}$.

Table 6 shows the comparison of the results for the tool paths of machining areas in Roughing, Finish Milling Bottom, and Finish Milling Corner when they are not optimized and optimized by the method in this paper. Compared with the non-optimized method, the total tool path length of Roughing and Finish Milling Bottom using the machining sequence optimization method is reduced by $2877.1 \mathrm{~mm}$ and $2448.1 \mathrm{~mm}$, respectively, and the efficiency has been increased by $21.3 \%$ and $17.1 \%$, respectively. After the machining areas are optimized under Finish Milling Contour, the tool path length is greatly reduced, and the machining efficiency is improved significantly. In general, the machining efficiency of the machining area under each working step has been improved to a certain extent after the machining sequence is optimized. Therefore, the machining sequence optimization method proposed in this article can effectively shorten the tool path length, thereby reducing machining time and improving machining efficiency.

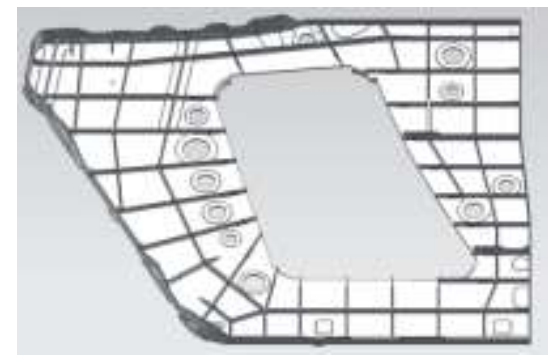

a Part3

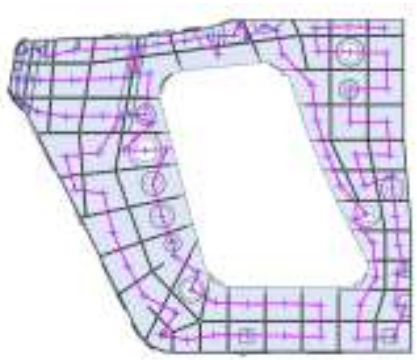

c Optimized tool path of Finish Milling Bottom

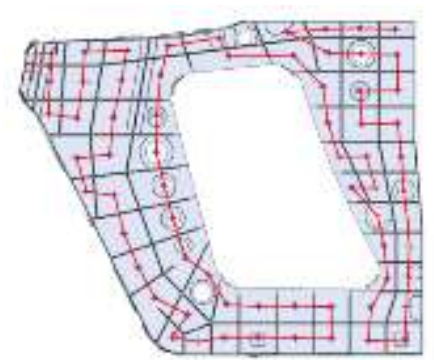

b Optimized tool path of Roughing

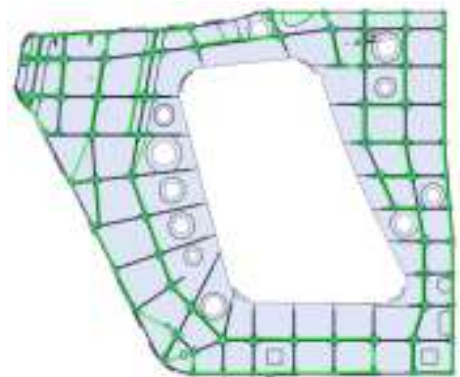

d Optimized tool path of Finish Milling Corner

Fig. 13 Machining sequence optimization case

Table 6 Comparisons between optimization approach and non-optimized approach

\begin{tabular}{|c|c|c|c|}
\hline \multirow{2}{*}{ Working step } & \multicolumn{2}{|c|}{ Length of tool path (mm) } & \multirow{2}{*}{$\begin{array}{l}\text { Improvement in } \\
\text { efficiency }\end{array}$} \\
\hline & optimization approach & non-optimized approach & \\
\hline Roughing & 10597.6 & 13474.7 & $21.3 \%$ \\
\hline $\begin{array}{l}\text { Finish Milling } \\
\text { Bottom }\end{array}$ & 11823.4 & 14271.5 & $17.1 \%$ \\
\hline $\begin{array}{l}\text { Finish Milling } \\
\text { Corner }\end{array}$ & 13257.6 & 22984.3 & $42.3 \%$ \\
\hline
\end{tabular}




\section{Conclusion and future work}

This paper proposes an efficient NC process scheme generation method based on reusable macro and micro process fusion. First, according to the calculation of the semantic distance of the process design intent, the micro processes are mapped to the macro process to realize the fusion of the macro process and the micro processes, and the compatibility credible evaluation model is established to evaluate the compatibility of the fusion result. Then, when the fusion result is credible, the machining area corresponding to the process scheme is optimized and adjusted from the geometrical level. The optimization adjustment of the machining area mainly realizes the merging of the machining area and the optimization of the machining sequence. Finally, actual parts are used for performance testing. The experimental results show that this method can improve design efficiency and machining efficiency.

The next step of the research work includes: (1) At present, the machining areas are mainly optimized and adjusted by the method; for other attributes (cutting tools, cutting parameters), optimization algorithms need to be further designed, such as considering the depth of cut to optimize the number of machining layers; (2) The current optimization goal is mainly machining time, and other optimization goals, such as energy consumption, can be considered in the future; this becomes a multi-objective optimization problem to obtain a high-efficiency and low-energy NC process scheme.

Availability of data and materials The raw/processed data required to reproduce these findings cannot be shared at this time as the data also forms part of an ongoing study.

Author contribution Bo Huang and Rui Huang conceived and designed the study. Xiuling Li performed the experiments. Bo Huang wrote the paper. Kai He, Feifei Zhang, and Shusheng Zhang reviewed and edited the manuscript. All authors read and approved the manuscript.

Funding This work is supported by the National Science Foundation of China (No. 52105518, 52105414, 52075148, 51875474).

\section{Declarations}

Ethical approval The article follows the guidelines of the Committee on Publication Ethics (COPE) and involves no studies on human or animal subjects.

Consent to participate Applicable.

Consent to publish Applicable.

Conflicts of interest/Competing interests The authors have no conflict of interests to disclose.

\section{References}

1. Yanhong Z, Baicun W. Toward New-Generation Intelligent Manufacturing[J]. Engineering, 2018, 4(1):11-20.

2. Liu Y, Xu X. Industry 4.0 and Cloud Manufacturing: A Comparative Analysis[J]. Journal of Manufacturing Science and Engineering, 2016, 139(3):034701.

3. Tao F, Qi Q, Liu A, et al. Data-driven smart manufacturing[J]. Journal of Manufacturing Systems, 2018, 48: 157-169.

4. Chen X, Gao S, Guo S, et al. A flexible assembly retrieval approach for model reuse [J]. Computer-Aided Design, 2012, 44(6):554-574.

5. Shahin A, Janatyan N. Group technology (GT) and lean production: a conceptual model for enhancing productivity. International Business Research, 2010, 3(4):105-118. 
6. Lee C S, Lee J H, Kim D S, et al. A hole-machining process planning system for marine engines [J]. Journal of manufacturing systems, 2013, 32(1): 114-123.

7. Zhang $X$, Nassehi A, Safaieh $M$, et al. Process comprehension for shopfloor manufacturing knowledge reuse [J]. International Journal of Production Research, 2013, 51(23-24): 7405-7419.

8. Zhang X, Nassehi A, Newman S T. Feature recognition from CNC part programs for milling operations [J]. International Journal of Advanced Manufacturing Technology, 2014, 70(1-4):397-412.

9. Zhou N, Liu X. Feature-based automatic NC programming for aero-engine casings [J]. Proceedings of the Institution of Mechanical Engineers, Part B: Journal of Engineering Manufacture, 2019, 233(4): 1289-1301.

10. Li Z, Zhou X, Liu W, et al. A geometry search approach in case-based tool reuse for mould manufacturing[J]. International Journal of Advanced Manufacturing Technology, 2015, 79(5-8):757-768

11. Liu J, Zhou H, Tian G, et al. Digital twin-based process reuse and evaluation approach for smart process planning. The International Journal of Advanced Manufacturing Technology, 2019, 100(5-8):1619-1634.

12. Jong W R, Lai P J , Chen Y W, et al. Automatic process planning of mold components with integration of feature recognition and group technology [J]. The International Journal of Advanced Manufacturing Technology, 2015, 78(5-8):807-824.

13. Zheng L Y, Dong H F, Vichare P, et al. Systematic modeling and reusing of process knowledge for rapid process configuration $[\mathrm{J}]$. Robotics and Computer-Integrated Manufacturing, 2008, 24(6):763-772.

14. Chen S, Zheng G, Zhou M, et al. Process-scheme-driven automatic construction of NC machining cell for aircraft structural parts [J]. Chinese Journal of Aeronautics, 2013, 26(5):1324-1335.

15. Li Y, Liu X, Gao J, et al. A dynamic feature information model for integrated manufacturing planning and optimization[J]. CIRP Annals - Manufacturing Technology, 2012, 61(1):167-170.

16. Huang $\mathrm{R}$, Zhang $\mathrm{S}$, Bai $\mathrm{X}$, et al. An effective subpart retrieval approach of $3 \mathrm{D}$ CAD models for manufacturing process reuse[J]. Computers in Industry, 2015, 67:38-53.

17. Huang R, Zhang S, Bai X, et al. An effective numerical control machining process reuse approach by merging feature similarity assessment and data mining for computer-aided manufacturing models[J]. Proceedings of the Institution of Mechanical Engineers, Part B: Journal of Engineering Manufacture, 2015, 229(7):1229-1242.

18. Xu C , Zhang S, Feng Z , et al. A multi-source similar subparts based $\mathrm{NC}$ process fusion and regrouping approach[J]. International Journal of Advanced Manufacturing Technology, 2019, 102: 185-199.

19. Pan W, Chen X, Gao S. Automatic shape adaptation for parametric solid models[J]. Computer-Aided Design, 2015, 62: 78-97.

20. Wang L, Song Y, Gao Q. Designing function blocks for distributed process planning and adaptive control [J]. Engineering Applications of Artificial Intelligence, 2009, 22(7):1127-1138. 
21. Wang L, Holm M, Adamson G. Embedding a process plan in function blocks for adaptive machining [J]. CIRP Annals - Manufacturing Technology, 2010, 59(1):433-436.

22. Wang L. An overview of function block enabled adaptive process planning for machining [J]. Journal of Manufacturing Systems, 2015, 35:10-25.

23. Wang W, Li Y, Ma Y. Towards a Feature-based Agent-driven NC Tool Path Generation to Support Design and Process Changes [J]. Computer-Aided Design and Applications, 2013, 10(4):603-618.

24. Liu C, Li Y, Gao X. Feature-based adaptive numerical control programming method for the environment of changing manufacturing resources [J]. Proceedings of the Institution of Mechanical Engineers Part B Journal of Engineering Manufacture, 2015, 230(8):237-247.

25. Xia Q, Etienne A, Dantan J Y, et al. Reconfigurable machining process planning for part variety in new manufacturing paradigms: definitions, models and framework [J]. Computers \& Industrial Engineering, 2018, 115:206-219.

26. Secer A, Sonmez A C, Aydin H. Ontology mapping using bipartite graph $[\mathrm{J}]$. International Journal of Physical Sciences, 2011, 6(17): 4224-4244.

27. Chen Z C, Fu Q. An optimal approach to multiple tool selection and their numerical control path generation for aggressive rough machining of pockets with free-form boundaries. Computer-Aided Design, 2011, 43(6):651-663.

28. Eppinger S D, Browning T R. Design structure matrix methods and applications[M]. MIT press, 2012. 